\title{
Search for unknown interaction with neutrons
}

\author{
Masaaki Kitaguchi* \\ Center for Experimental Studies, KMI, Nagoya University \\ E-mail: kitaguchi@phi.phys.nagoya-u.ac.jp
}

\begin{abstract}
Neutron is a chargeless massive particle with the lifetime in the macroscopic range, which is suitable for precision measurement of the small influence of new physics. We have started the experimental studies to search unknown interactions with slow neutrons. Combination of the pulsed neutrons provided by J-PARC and the advanced optical devices enables us to perform new types of high precision measurements. The unknown source of large $\mathrm{CP}$ violation should exist to explain the matter universe. One of the strongest constraint of the search for the large CP-violation is the result of neutron electric dipole moment. We are now discussing another approach to search neutron EDM by using dynamical diffraction of neutron wave with non-centrosymmetric crystals. The enhancement of the violation of time-reversal symmetry is predicted in the neutron capture reaction for some nuclei. We have also started the feasibility to search the T-violation with the neutron resonance capture reaction at J-PARC. Non-Newtonian effect of the gravity at the short range is lead by the existence of extra-dimension of the space. Neutron scattering with noble gas target enables us to measure the interaction at the range of the order of $1 \mathrm{~nm}$. On the other hand, we are now planning the unknown force search lead by dark energy, like chameleon field, by using the interferometer.
\end{abstract}

The 3rd International Symposium on "Quest for the Origin of Particles and the Universe" 5-7 January 2017

Nagoya University, Japan

${ }^{*}$ Speaker. 


\section{Introduction}

Neutron is a chargeless massive particle with the lifetime in the macroscopic range, which is suitable for precision measurement of the small influence of new physics. We have started the experimental studies to search unknown interactions with slow neutrons. Combination of the pulsed neutrons provided by J-PARC and the advanced optical devices enables us to perform new types of high precision measurements. In this paper, four topics of our research project are reported. The two are about CP-violation search, and the others are about exotic interaction.

\section{CP violation}

\subsection{Search for T-violating effect in resonance capture reaction}

The large charge-parity $(\mathrm{CP})$ symmetry violation is required to explain the today's matter universe. In order to discuss new physics beyond the standard model of particle, the search for the large $\mathrm{CP}$-violation is quite important.

The large enhancement of parity $(\mathrm{P})$ violation of the weak interaction contained in the nuclear interaction was discovered in 2000's [1]. The asymmetry of the capture cross section with respect to the helicity of incident neutrons was enhanced by at most $10^{6}$ times larger in a several nuclei. The enhancement is explained as the result of the entrance channel interference between neighboring s-wave and p-wave amplitudes. The mechanism of the P-violating effect has been proposed theoretically to be applicable to enhance the experimental sensitivity to search the time-reversal (T) violation effect in the p-wave resonances [2]. The advantage to apply the enhancement mechanism is that takes T-odd effects due to the final-state interaction is expected to be negligibly small as the neutron propagation through the target material can be described by the neutron optics. The enhancement of T-violation is given as

$$
\Delta \sigma_{\mathrm{CP}}=\kappa(J) \frac{W_{\mathrm{T}}}{W} \Delta \sigma_{\mathrm{P}}
$$

where $\Delta \sigma_{\mathrm{CP}}$ and $\Delta \sigma_{\mathrm{P}}$ are the CP-violating and P-violating cross section, $\kappa(J)$ is the angularmomentum factor, $W_{\mathrm{T}}$ and $W$ denote the $\mathrm{CP}$ - and P-violating matrix elements. This means that the candidate nuclei for T-violation search are the nuclei with large enhancement of P-violation, however, the parameter $\kappa(J)$ must be measured. The angular-momentum factor $\kappa(J)$ can be written with the partial neutron widths of p-wave resonance. When we assume s-p mixing, the partial neutron width can be measured by the angular distribution of individual $\gamma$-rays from the compound resonance [3]. This measurement requires that both the spin of the compound state $(J)$ and the spin of the final state of the $\gamma$-ray transition are known.

Combination of the intense pulsed neutrons and the germanium detectors with high energy resolution enables us to perform this kind of measurements. We are now performing the experiments at the beamline BL04 ANNRI in J-PARC. The angular distribution of resonance capture reaction is measured with $4 \pi$ germanium spectrometer. Target nuclei were ${ }^{139} \mathrm{La},{ }^{131} \mathrm{Xe},{ }^{115} \mathrm{In}$, and so on, which were the nuclei with large enhancements of P-violation. The resonance parameters of the compound nuclei has been measured with very high energy resolution. The angular distributions of the individual $\gamma$-rays has been also measured. Detailed analysis is now in progress. 
To measure the T-odd correlation term, both of neutron polarization and target polarization techniques are required (Fig. 1). Helium and/or proton spin filter are discussed as a neutron polarizer. We are also discussing the polarization of the target nuclei by using spin exchange optical pumping (SEOP) and/or dynamical nuclear polarization (DNP). Research and development of High speed neutron detector are required to measure the small asymmetry of capture cross section of the order of $10^{-6}$. We have started the development of the detector with liquid scintillator. We emphasize that the series of existing technique can be applied for the T-violation search experiments. For example, we are discussing the feasibility of the experiment with polarized Xe target in J-PARC (Fig. 2).

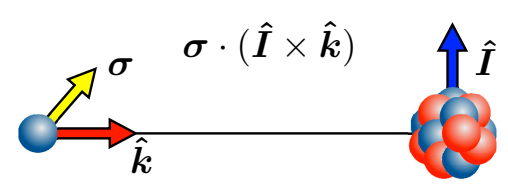

Figure 1: Schematic view of T-violating correlation in the neutron-nuclei reaction.

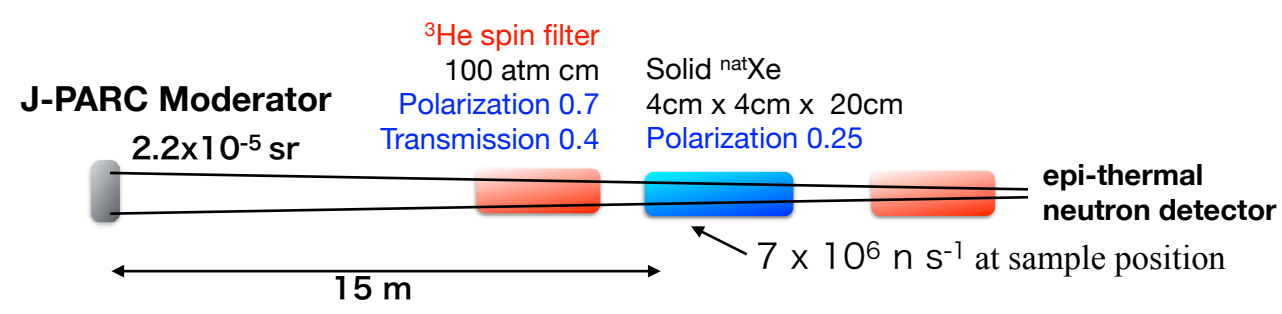

Figure 2: Experimental design for T-violation search at J-PARC.

\subsection{EDM search with crystal diffraction}

Non-zero value of the permanent electric dipole moment of neutrons (nEDM) signals the violation of time-reversal $(\mathrm{T})$ invariance. Although experimental searches have been pursued in the world, the nEDM has not yet been observed. The present upper limit is $\left|d_{\mathrm{n}}\right|<3 \times 10^{-26} e \cdot \mathrm{cm}(90 \%$ C.L.), which is very close to the predictions of new physics beyond the standard model of particle physics, for example, supersymmetry. The most stringent upper limit of the nEDM measurement was obtained in the spin precession frequency in both magnetic field and electric field of confined ultra-cold neutrons (UCN method) [4]. Although next-generation UCN sources are being developed intensively at accelerator-based neutron facilities for improving the upper limit with the UCN method $[5,6,7,8]$, it is valuable and very important to develop different methods to measure the $\mathrm{nEDM}$ for confirming the present upper limit with different systematic uncertainties and hopefully improve the experimental sensitivity.

The sensitivity of nEDM search written as

$$
\Delta d_{\mathrm{n}} \propto \frac{1}{E t \sqrt{N}},
$$

where $N$ is the number of neutrons, $E$ is the applied electric field, and $t$ is the interaction time. The high sensitivity of the UCN method is achieved with the extremely large value of $t$. 
On the other hand, some types of non-centrosymmetric crystals have strong effective electric field inside their lattice. Although the interaction time of the cold neutrons which pass through the crystal is shorter than that of UCNs, the achieved sensitivity can be competitive because of the strong electric field and more intense incident flux. Furthermore, this method has a potential to improve the sensitivity if we successfully prepare a crystal which has much stronger electric field and has a large dimension. The nEDM experiments with quartz crystals have been already reported [9]. The effective electric field was experimentally measured to be $E=2 \times 10^{8} \mathrm{~V} / \mathrm{cm}$. The electric field can be increased by about an order of magnitude by employing bismuth germanate, lead oxide, $\cdots$, instead of quartz. The electric field in the bismuth germanate crystal is estimated as $10^{9} \mathrm{~V} / \mathrm{cm}$ [10]. We have started to study the feasibility of EDM measurement with Bismuth Germanate $\left(\mathrm{Bi}_{12} \mathrm{GeO}_{20}\right)$ crystal.

Neutrons with the proper incident angle are reflected and transmitted in the crystal according to Bragg's law. On the entry to the crystal, both waves are refracted due to the Fermi pseudopotential. In the case of the periodic boundary condition of the single crystal, the propagation of each wave can be written as a superposition of two Bloch functions. The result of the interference between the waves makes the propagation of standing wave along to the crystal lattice (Fig. 3). This wave feels the strong effective electric field in the crystal, which makes precession of electric dipole moment. The effective electric field can be measured by using this dynamical diffraction effects. The spin rotation in the Schwinger magnetic field, which is induced by passing neutron magnetic moment in the electric field, is measured. We are now trying to measure the electric field of BGO at the polarized neutron beamline BL17 Sharaku in J-PARC.

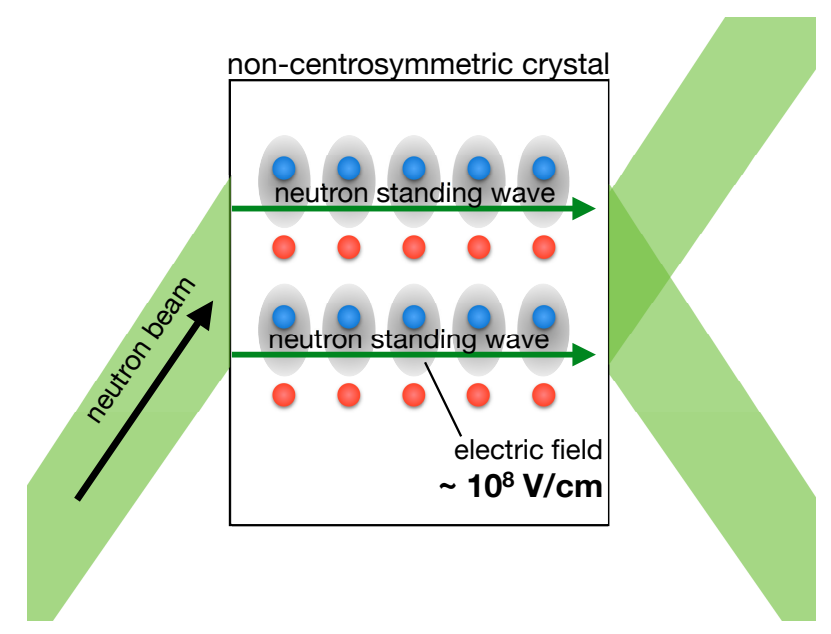

Figure 3: Neutron wave propagation according to dynamical diffraction in non-centrosymmetric crystal.

\section{Intermediate-range force}

\subsection{Short-range force search with gas scattering}

Some models beyond the standard model predict the existence of non-Newtonian gravity [11, 12, 13]. A new possible force which couples to mass can be seen at short-range. Generally, the 
unknown potential $V$ is written as Yukawa-type potential like

$$
V(r)=-\frac{G_{N} m_{1} m_{2} \alpha}{r} \exp \left(-\frac{r}{\lambda}\right)
$$

where $G_{N}$ is the gravitational constant, $m_{1}, m_{2}$ are masses of two particles, $\lambda$ is a Compton wave length of new boson and $\alpha$ is a coupling constant. The constraints above $10 \mathrm{~nm}$ were measured by using torsion balances or isoelectronic techniques $[14,15,16]$. In the range below $10 \mathrm{~nm}$, neutron scattering with atoms has the advantage to make a constraint because the electromagnetic interaction and Van der Waals force are suppressed [17, 18]. Scattering of slow neutron with atom can be explained by using Born approximation. The existence of the Yukawa-type force causes the forward scattering distribution, although the nuclear interaction makes isotropic scattering. The measurement of the angular distribution of the scattering enables us to search the unknown shortrange force. We are now performing the neutron scattering experiment at J-PARC.

The apparatus was installed into beamline BL05 NOP. The schematic view of the experimental setup is shown in figure 4. For the target, we used the noble gases because they don't have molecular or crystal structure, atomic spin. In order to estimate the effect of gas pressure and mass dependence of the target, we used helium, argon, and xenon gas for the targets. The thin aluminum plates were utilized for the windows of the gas cells to reduce the scattering. ${ }^{3} \mathrm{He}$ two dimensional position sensitive detector was used to measure the scattering distribution. The first physics run was performed in June 2016. We are also developing the Monte-Carlo simulation to calculate the scattering distribution including finite volume of the gas cell, gas thermal motion, and the other effects precisely. The analysis by comparing the data with the simulations are on going.

We are planning to improve our statistics by increasing the Xe gas pressure in the gas cell and using a larger beam size to update the sensitivity.

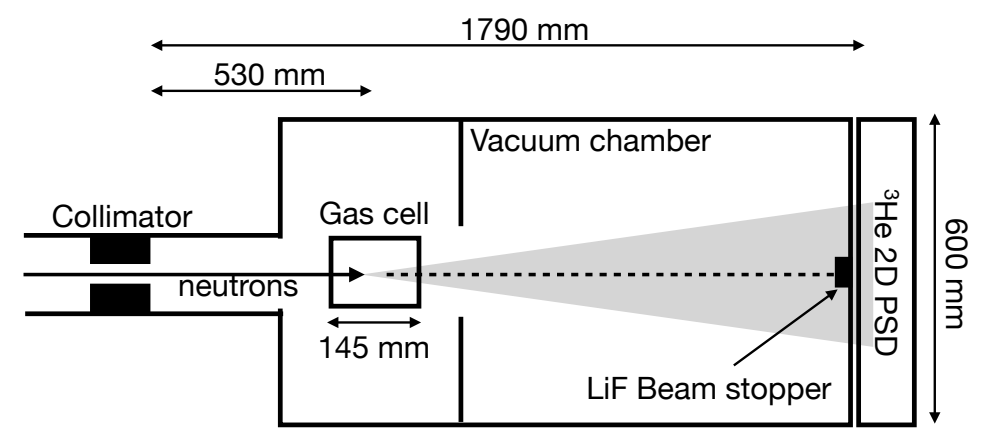

Figure 4: The setup of gas scattering experiment at BL05 NOP in J-PARC.

\subsection{Dark energy search with neutron interferometer}

The accelerating expansion of the universe suggests that the unknown energy called as dark energy exists $[19,20,21]$. One of the candidates of the dark energy is the chameleon mechanism, which modify the gravity in the presence of the matter density [22]. This means that the gravitational potential should change near the matter.

Neutron interferometer has the advantage to measure the gravitational potential precisely (Fig. 5). The change of the potential results the phase shift of the interferogram. The experimental 
searches was performed by using small neutron interferometer, there was no evidence for the chameleon dark energy. The sensitivity depends on the interaction length inside the interferometer. Large scale of silicon perfect crystal ingots are prepared for this experiment. We fabricated a small piece of interferometer for test the ingots. The test experiments are now started at Neutron Optics and Interferometry Facility (NIOF) in NIST.

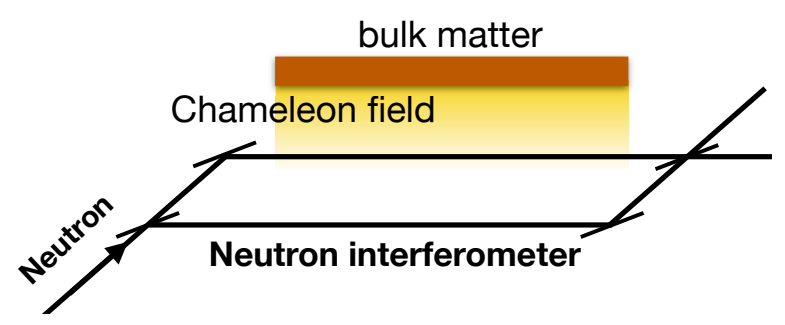

Figure 5: Schematic view of chameleon search with neutron interferometer.

\section{Conclusion}

Neutron is suitable for precision measurement to search new physics. Combination of the intense pulsed neutrons and the advanced optical devices enables us to perform new types of high precision measurements. The large enhancement of T-violating effect in compound nuclei can be one of the powerful techniques to search CP-violation. The crystal diffraction method is another way to measure the nEDM with different systematic uncertainties. Gas scattering experiment and neutron interferometer have the advantage to study gravity-like unknown forces. Research and development for each experiment have started in J-PARC.

\section{References}

[1] G. E. Mitchell, Physics Reports 354, 157?241 (2001).

[2] V. P. Gudkov, Phys. Rep. 212, 77 (1992).

[3] V.V. Flambaum, O.P. Sushkov, Nucl. Phys. A 412, 13 (1984).

[4] C.A. Baker, et al., Phys. Rev. Lett. 97131801 (2006).

[5] A. Saunders, et al., Phys. Lett. B 59355 (2004).

[6] A. Anghel, et al., Nucl. Instr. and Meth. A 611272 (2009).

[7] Y. Masuda, et al., Phys. Rev. Lett. 89284801 (2002).

[8] N. Fomin, et. al., Nucl. Instr. and Meth. A 773, 45-51 (2015).

[9] V.V. Fedorov, et. al., Physica B: Condensed Matter 297, 293 (2001).

[10] V.L. Alexeev, et al., Nucl. Instr. and Meth. A, 284, 181 (1989).

[11] Nima Arkani-Hamed, Savas Dimopoulos, and Gia Dvali, Phys. Lett. B 429, 2632272 (1998).

[12] Nima Arkani-Hamed, Savas Dimopoulos, and Gia Dvali., Phys. Rev. D 59, 086004 (1999).

[13] Y. fujii, Nature Physical Science, 234, 5 ?7 (1971). 
[14] E. Fischbach, et. al., Phys. Rev. D 64, 075010 (2001).

[15] M. Bordag, et. al., Phys. Rep. 353, 1 ?205 (2001).

[16] R. S. Decca, et. al., Phys. Rev. Lett. 94, 240401 (2005).

[17] V. V. Nesvizhevsky, et. al., Phys. Rev. D 77, 034020 (2008).

[18] Y. Kamiya, et. al., Phys. Rev. Lett. 114, 161101 (2015).

[19] J.A. Frieman, M.S. Turner, and D. Huterer, Annu. Rev. Astron. Astrophys. 46, 385 (2008).

[20] B. Ratra and P. J. E. Peebles, Phys. Rev. D 37, 3406 (1988).

[21] R. R. Caldwell, R. Dave, and P. J. Steinhardt, Phys. Rev. Lett. 80, 1582 (1998).

[22] J. Khoury and A. Weltman, Phys. Rev. Lett. 93, 171104 (2004).

[23] H. Lemmel et al. Phys. Lett. B 743, 310 (2015). 